\title{
IN.PACT Amphirion paclitaxel eluting balloon versus standard percutaneous transluminal angioplasty for infrapopliteal revascularization of critical limb ischemia: rationale and protocol for an ongoing randomized controlled trial
}

Thomas Zeller $^{1 *}$, Iris Baumgartner ${ }^{2}$, Dierk Scheinert ${ }^{3}$, Marianne Brodmann $^{4}$, Marc Bosiers ${ }^{5}$, Antonio Micari ${ }^{6}$, Patrick Peeters ${ }^{7}$, Frank Vermassen ${ }^{8}$, and Mario Landini ${ }^{9}$ on behalf of the IN.PACT DEEP Trial Investigators

\begin{abstract}
Background: The effectiveness and durability of endovascular revascularization therapies for chronic critical limb ischemia (CLI) are challenged by the extensive burden of infrapopliteal arterial disease and lesion-related characteristics (e.g., severe calcification, chronic total occlusions), which frequently result in poor clinical outcomes. While infrapopliteal vessel patency directly affects pain relief and wound healing, sustained patency and extravascular care both contribute to the ultimate "patient-centric" outcomes of functional limb preservation, mobility and quality of life (QoL).

Methods/Design: IN.PACT DEEP is a 2:1 randomized controlled trial designed to assess the efficacy and safety of infrapopliteal arterial revascularization between the IN.PACT Amphirion ${ }^{\mathrm{TM}}$ paclitaxel drug-eluting balloon (IA-DEB) and standard balloon angioplasty (PTA) in patients with Rutherford Class 4-5-6 CLI.
\end{abstract}

Discussion: This multicenter trial has enrolled 358 patients at 13 European centers with independent angiographic core lab adjudication of the primary efficacy endpoint of target lesion late luminal loss (LLL) and clinically driven target lesion revascularization (TLR) in major amputation-free surviving patients through 12-months. An independent wound core lab will evaluate all ischemic wounds to assess the extent of healing and time to healing at 1, 6, and 12 months. A QoL questionnaire including a pain scale will assess changes from baseline scores through 12 months. A Clinical Events Committee and Data Safety Monitoring Board will adjudicate the composite primary safety endpoints of all-cause death, major amputation, and clinically driven TLR at 6 months and other trial endpoints and supervise patient safety throughout the study. All patients will be followed for 5 years. A literature review is presented of the current status of endovascular treatment of CLI with drug-eluting balloon and standard PTA. The rationale and design of the IN.PACT DEEP Trial are discussed. IN.PACT DEEP is a milestone, prospective, randomized, robust, independent core lab-adjudicated CLI trial that will evaluate the role of a new infrapopliteal revascularization technology, the IA-DEB, compared to PTA. It will assess the overall impact on infrapopliteal artery patency, limb salvage, wound healing, pain control, QoL, and patient mobility. The 1-year results of the adjudicated co-primary and secondary endpoints will be available in 2014.

Trial registration: NCT00941733

Keywords: Peripheral vascular disease, Critical limb ischemia, Infrapopliteal, Drug-eluting balloon

\footnotetext{
*Correspondence: Thomas.Zeller@universitaets-herzzentrum.de

'Department of Angiology, Universitäts Herzzentrum Freiburg Bad Krozingen,

Bad Krozingen, Germany

Full list of author information is available at the end of the article
} 


\section{Background}

Percutaneous transluminal angioplasty (PTA) is an established alternative to open surgical bypass for the treatment of infrainginual disease of critical limb ischemia (CLI) patients with limited life expectancy, multiple surgical comorbidities, and/or those who lack an adequate venous conduit [1-3]. Several studies established lower peri-procedural mortality and morbidity and high technical success for PTA, leading some centers to consider infrapopliteal artery angioplasty, particularly in specific high-risk CLI patient cohorts, to be the "first line" therapeutic approach [4-7]. Conversely, the extent and burden of infrapopliteal arterial disease, particularly in diabetics, are well described [8], and high post-PTA restenosis rates have been consistently reported [9-11]. Nonetheless, reports that include adjudication of restenosis rates by an independent angiographic core lab as part of a large multicenter randomized controlled trial are lacking. Likewise, the impact of high infrapopliteal vessel restenosis rates on the prognosis of CLI patients is unclear. Yet, despite concerns of high infrapopliteal PTA restenosis rates, the limb salvage rates appear acceptable [10] when compared to open lower limb bypass [12]. Thus, there is an evolving impression, endorsed by societal guidelines [13,14], that the potential near-term benefit of the less invasive PTA approach in CLI patients, when compared to surgery, favors PTA as the "first line approach" in specific patients. The potential negative impact on limb salvage of a required percutaneous reintervention or surgical bypass after a failed PTA remains, however, a concern. Recent data suggest that patients who experienced tibial PTA restenosis and who subsequently underwent a lower limb bypass required more distal target anastomoses [15] and had higher 1-year amputation and graft closure rates [16] when compared to patients without prior PTA. Likewise, the BASIL trial investigators observed that patients undergoing bypass after a failed PTA had worse amputation-free survival (AFS) rates than those undergoing primary bypass [3]. Therefore, there is an evolving concern that CLI patients followed for a longer time period will demonstrate high tibial PTA restenosis rates that may ultimately require lower extremity bypass, which are, in turn, associated with higher bypass failure rates and worse outcomes [16].

Paclitaxel drug-eluting balloons are designed to promote arterial patency by reducing neointimal proliferation. In prospective clinical trials in relatively small CLI patient cohorts, tibial vessel drug-eluting balloon (DEB) angioplasty was associated with significantly reduced restenosis rates and late lumen loss (LLL) at 3, 6, and 12 months [17-19]. Although these angiographic assessments were not core lab adjudicated, there is a growing perception that the clinical results of DEB tibial angioplasty mirror the clinical experience in the superficial femoral artery in claudicants [20-23]. However, the potential for tibial artery DEB angioplasty to provide a more durable patency rate and, thereby, potentially favorably impact limb salvage, wound healing, time to wound healing, pain control, quality of life (QoL) indices, and mobility has yet to be defined in a prospective, robust, randomized assessment compared to PTA. IN.PACT DEEP was initiated as the first trial to systemically assess the Safety and Efficacy of the new IN.PACT Amphirion $^{\text {Th }}$ paclitaxel drug-eluting balloon (IA-DEB) technology in CLI patients with infrapopliteal disease.

\section{Hypothesis}

We hypothesize that the IA-DEB will significantly reduce angiographically assessed target lesion late lumen loss (LLL) compared to standard PTA in infrapopliteal lesions up to $10 \mathrm{~cm}$ in length and reduce clinically driven target lesion revascularization (TLR) in amputation-free surviving CLI patients through 12 months. Moreover, we assumed that the IA-DEB would be non-inferior to PTA with reference to the rate of allcause death, major amputation, and clinically driven TLR through 6 months.

\section{Methods/Design}

\section{Trial design}

This trial is a 2:1 randomized, controlled, patient-blinded multicenter trial that compares the IA-DEB to PTA in terms of angiographically assessed target lesion LLL and clinically driven TLR of infrapopliteal arteries in Rutherford class 4-5-6 symptomatic critical limb ischemia patients through 12 months (Figure 1). Consecutive patients matching all General Eligibility Criteria (Table 1 Sections A-D) were consented for the trial. All patients were required to be free of further general procedural exclusion criteria (Table 1 Section E) such as unsuccessful crossing of the target lesion with a guide wire.

This trial is composed of two subject cohorts (Clinical cohort and Angio cohort), the second characterized by more restrictive eligibility criteria than the first: subjects who met certain additional and specific eligibility criteria and avoided specific exclusion criteria (Table 1 Sections F and G) were allocated to an "Angio" cohort; the others were allocated to the "Clinical" cohort. Finally, subjects were randomized 2:1 to IA-DEB or PTA within their assigned cohort. All subjects from both cohorts will be part of the clinical assessment and followed for 5 years post-randomization. Only subjects from the Angio cohort will undergo angiographic evaluation at 12 months. In August 2012, the IN.PACT DEEP Trial completed enrollment of 358 subjects (Figure 1).

\section{Primary efficacy endpoints}

The IN.PACT DEEP trial was designed before there was preliminary evidence of the degree of efficacy of DEB 


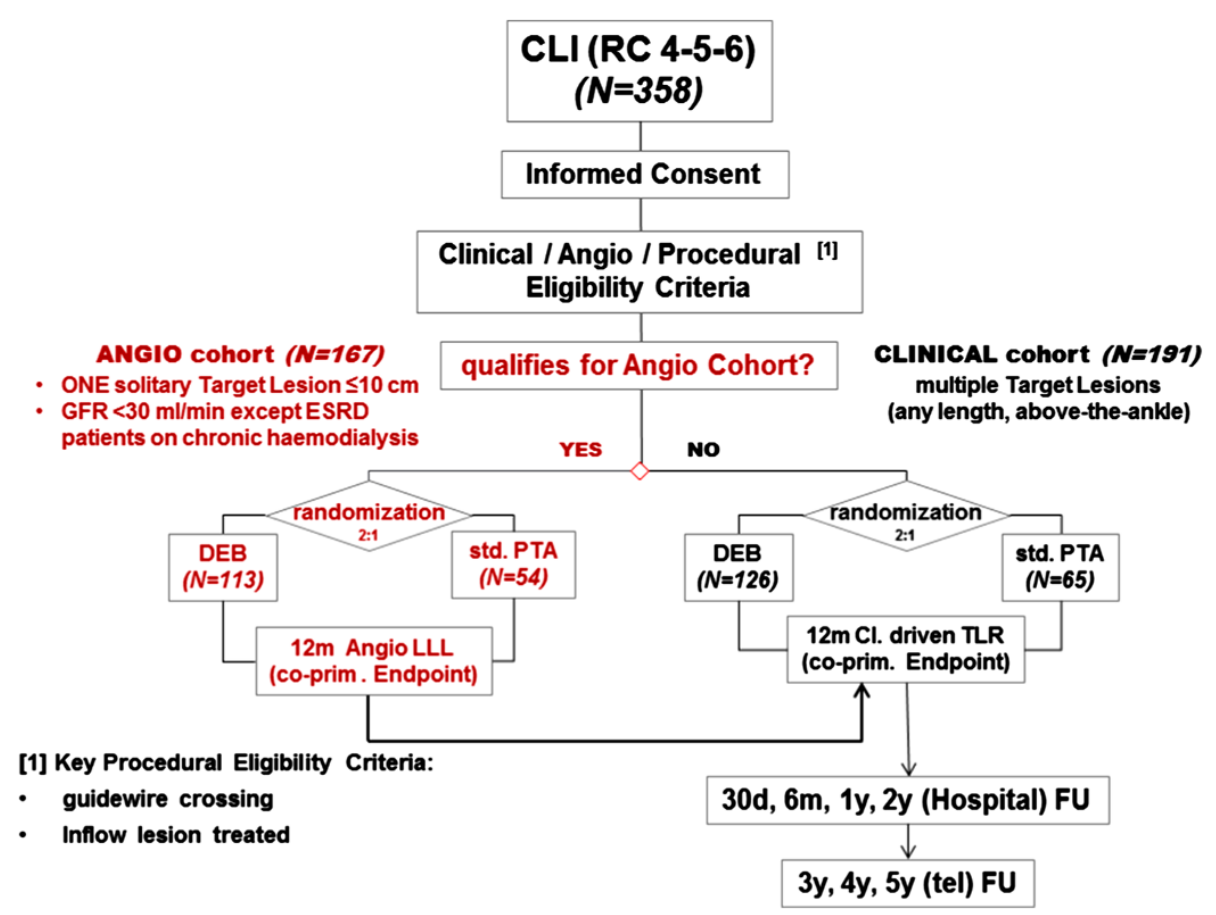

Figure 1 IN.PACT DEEP treatment cohort assignment/randomization flowchart.

relative to PTA in the infrapopliteal vasculature. It was therefore decided to choose a method capable of distinguishing even subtle differences in performance. LLL assessment is considered the most sensitive, objective determinant of post-intervention vessel narrowing; this methodology was accordingly selected as the best arbiter of a potential DEB treatment effect. Thus, an angiographic core lab-adjudicated infrapopliteal artery target lesion LLL at 12 months post-intervention (applying to the Angio cohort only) and clinically driven TLR through 12 months (applying to the entire subject population) were selected as co-primary endpoints to provide assessment of vessel patency in the two treatment cohorts from both angiographic and clinical perspectives. "Clinically driven" TLR is defined as (1) directed by an increase in size of a pre-existing wound(s) and/or (2) occurrence of a new wound(s), and/or (3) deterioration in Rutherford class. To assist in quantitation of wound healing assessments, all investigational sites were provided with and trained on the use an electronic wound reader able to objectively record the morphological status of wound's area and depth (SilhouetteMobile ${ }^{\mathrm{TM}}$, Aranz Medical Ltd., Auckland, New Zealand). An independent Wound Core Lab. (SynvaCor, Springfield, IL) subsequently adjudicated data. USA. Important "patient-centric" secondary endpoints to be assessed include time to wound healing, change in ischemic pain, quality of life (QoL), and walking capacity. Other secondary endpoints include amputation-free survival (AFS), major adverse events (MAE) and sustained clinical improvement. The primary safety endpoint is described below; secondary endpoints and major inclusion/exclusion clinical and angiographic criteria are detailed in Tables 1 and 2, respectively.

\section{Subjects}

A total of 358 patients with symptomatic Rutherford class 4-5-6 CLI who matched the trial eligibility criteria were randomized 2:1 to treatment with IA-DEB $(N=$ $239)$ or PTA $(N=119)$. One hundred sixty-seven of these subjects were allocated to the Angio cohort (113 subsequently randomized to IA-DEB and 54 to PTA) and the remaining 191 to the Clinical cohort (126 subsequently randomized to IA-DEB and 65 to PTA). LLL angiography will be assessed at the 12-month follow-up for the Angio cohort only and clinically driven TLR through 12 months will be assessed in all subjects. All subjects will be evaluated yearly for 5 years.

\section{Eligibility criteria}

Subjects were required to meet all general, angiographic and procedural eligibility criteria (Table $1 \mathrm{~A}-\mathrm{E}$; see below) to be considered for the trial. If any of the exclusion criteria (Table $1 \mathrm{~B}$ and $\mathrm{E}$ ) were met, the subject was excluded from the trial.

Subjects who met certain specific additional inclusion and exclusion criteria (see Figure 1 and Table $1 F$ and G) were allocated to the Angio cohort. A maximum lesion 


\section{Table 1 IN.PACT DEEP inclusion and exclusion criteria}

(A) General inclusion criteria

i. 1 Age $\geq 18$ years and $\leq 85$ years

i.2 Patient or patient's legal representative has been informed of the nature of the study, agrees to participate, and has signed an ECapproved consent form

i.3 Female patients of childbearing potential have a negative pregnancy test $\leq 7$ days before the procedure and are willing to use a reliable method of birth control for the duration of study participation

i.4 Patient has documented chronic critical limb ischemia (CLI) in the target limb prior to the study procedure with Rutherford category 4, 5 , or 6

i.5 Life expectancy $>1$ year in the investigator's opinion

(B) General exclusion criteria

e.1 Patient unwilling or unlikely to comply with follow-up schedule

e.2 Planned major index limb amputation

(C) General angiographic inclusion criteria

i.6 Reference vessel diameter(s) between 2 and $4 \mathrm{~mm}$

i.7 Single or multiple lesions with $\geq 70 \%$ DS of different lengths in one or more main afferent crural vessels including tibioperoneal trunk

i.8 At least one non-occluded crural vessel with angiographically documented run-off to the foot either directly or through collaterals

(D) General angiographic exclusion criteria

e.3 Lesion and/or occlusions located in or extending to the popliteal artery or below the ankle joint space

e.4 Inflow lesion or occlusion in the ipsilateral iliac, SFA, or popliteal arteries with length $\geq 15 \mathrm{~cm}$

e. 5 Significant ( $\geq 50 \%$ DS) inflow lesion or occlusion in the ipsilateral iliac, SFA, or popliteal arteries left untreated

e.6 Previously implanted stent in the $\mathrm{TL}(\mathrm{s})$

e.7 Aneurysm in the target vessel

e.8 Acute thrombus in the $\mathrm{TL}$

(E) General procedural exclusion criteria

e.9 Failure to obtain $<30 \%$ residual stenosis in pre-existing, hemodynamically significant ( $\geq 50 \%$ DS and $<15 \mathrm{~cm}$ length) inflow lesions in the ipsilateral iliac, SFA, or popliteal artery. DES and/or DEB was not allowed for the treatment of inflow lesions

e.10 Failure to cross the $T L$ with a $0.014^{\prime}$ guide wire

e.11 Use of alternative therapy, e.g., atherectomy, cutting balloon, laser, radiation therapy, DES as part of the index procedure

(F) Angiographic cohort angiographic inclusion criteria

a.i.1 Angio-TL is one identifiable single solitary or a series of multiple adjacent lesions with a DS $\geq 70 \%$ and a cumulative length $\leq 100 \mathrm{~mm}$ that can be covered by a single IN.PACT Amphiron ${ }^{\text {TM }}$ (10-mm balloon landing zone in both edges is mandatory)

a.i.2 Angio-TL is the only lesion in that vessel (only 1 Angio- $T L$ per patient is allowed)

(G) Angiographic cohort general exclusion criteria

a.e.1 GFR <30 ml/min except for patients with renal end-stage disease on chronic hemodialysis length of $10 \mathrm{~cm}$ was selected for this cohort to facilitate the technical assessment of LLL by the blinded core lab.

\section{Extent of infrapopliteal revascularization and wound healing}

The angiosome concept, introduced by Taylor et al. [24], is an anatomic unit of tissue, fascia, muscle, and bone fed by a source artery and drained by specific veins. The lower extremity and foot are divided into six specific angiosomes fed by the posterior tibial artery ( 3 angiosomes), anterior tibial artery (1 angiosome), and peroneal artery ( 2 angiosomes). Alterations in regional pedal circulation impact successful healing in patients with ischemic ulcers $[25,26]$. Regional perfusion differences may be accentuated if the source or woundrelated artery (WRA) to the ischemic angiosome is occluded and collateral vessels perfuse the impacted angiosome indirectly.

Various contributing factors that influence successful revascularization and impact wound healing have been described. Iida [25] and Neville [27] demonstrated significantly improved wound healing and rates of freedom from amputation when direct pulsatile flow via the WRA was established compared to when indirect revascularization was implemented. Peregrin [28] observed that better outcomes were achieved with revascularization of a greater number of vessels, while Faglia [29] reported that perfusion via the anterior and/or posterior tibial arteries generally resulted in better outcomes when compared to peroneal artery recanalization alone. Still better outcomes were achieved with revascularization of a greater number of vessels. Additionally, the importance of foot vascular anatomy [30], pedal circulation [31], and micro-circulation [32] to CLI outcomes has been recently emphasized. Ultimately, the revascularization target and extent of the infrapopliteal/pedal revascularization remain an area of great interest and debate in CLI treatment. To address these issues, the IN.PACT DEEP trial will evaluate the impact of direct vs. indirect revascularization and the number of revascularized vessels on wound healing and limb salvage. This prospective evaluation consented subjects prior to the confirmation of full eligibility, which included some angiographic and procedural criteria. Subjects who failed angiographic and procedural eligibility criteria were considered "screen failures". The screen failures were not considered as enrolled subjects for the purposes of intent-to-treat (ITT) analysis.

\section{Randomization}

Randomization of subjects proceeded after all procedural and angiographic eligibility criteria had been met, including the requirements that all inflow lesions had been successfully treated and that the guidewire had 
Table 2 IN.PACT DEEP trial secondary endpoints

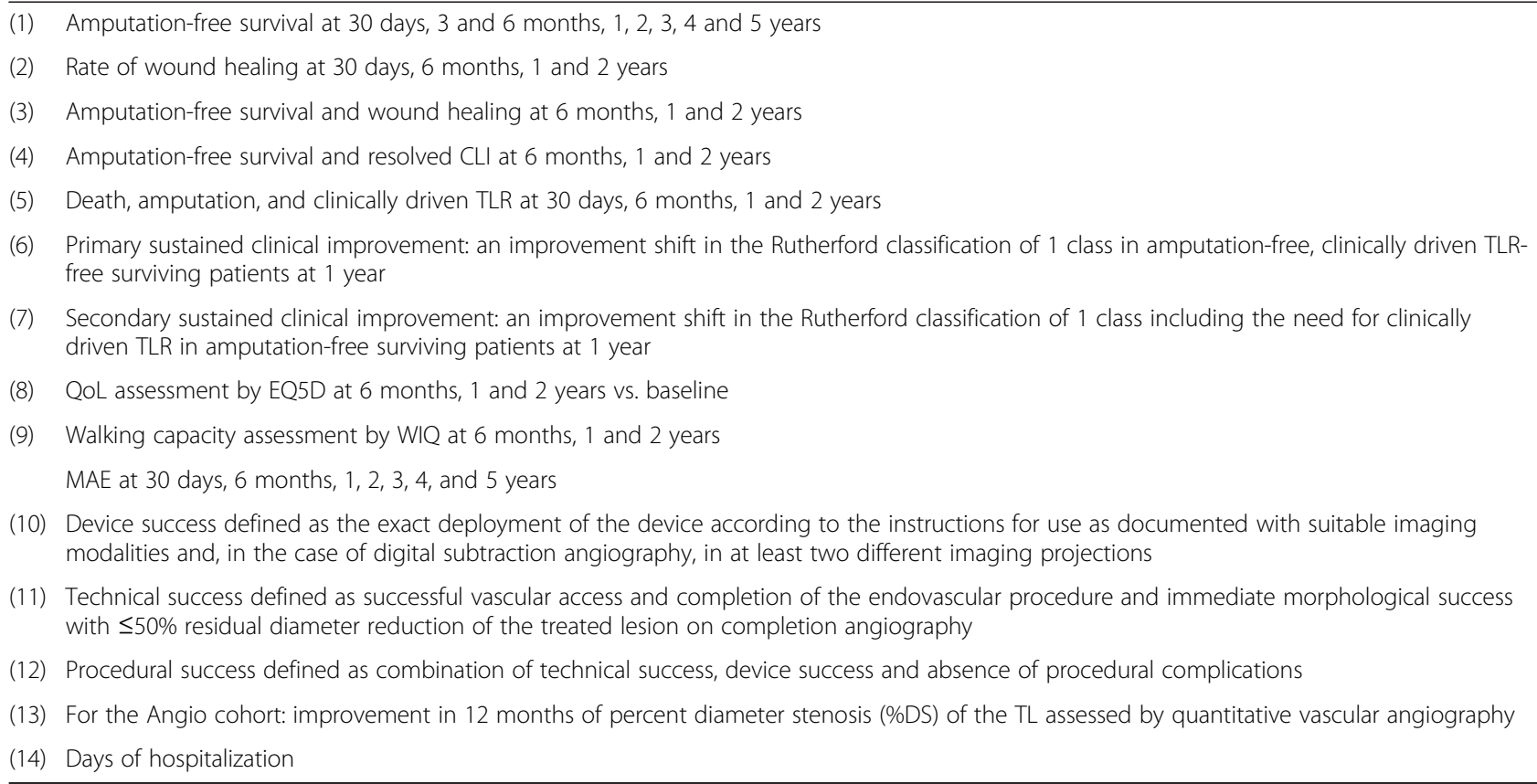

successfully crossed the target lesion and was positioned into the distal true lumen (Table 1). The randomization process was performed using blocks of sealed envelopes.

\section{Ethics}

This trial is conducted in accordance with the principles of the Declaration of Helsinki, ISO 14155 and Good Clinical Practices guidelines. The Ethics Committees of all investigational sites (Table 3) approved the trial protocol, and written informed consent was obtained from all subjects before enrollment. Subjects and their treating physicians retained the right to withdraw from the trial and all follow-ups at any time without prejudice.

\section{Safety and quality control Data safety monitoring board}

A Clinical Events Committee (CEC) will assess the composite primary safety endpoints of all-cause death, major amputation, and clinically driven TLR through 12 months and will adjudicate other trial endpoints. A Data Safety Monitoring Board (DSMB) will periodically

\section{Table 3 Ethics committees used in the IN.PACT DEEP trial}

\begin{tabular}{|c|c|}
\hline Site name & Ethics committees \\
\hline $\begin{array}{l}\text { Park-Krankenhaus Leipzig-Südost GmbH/Herzzentrum Leipzig } \\
\text { GmbH }\end{array}$ & Ethik Kommission, Härtelstrasse 16-18, 04107 Leipzig \\
\hline \multicolumn{2}{|l|}{ Medical Care Center Prof. Mathey, Prof. Schofer GmbH } \\
\hline University of Heidelberg & $\begin{array}{l}\text { FEKI (Prof H.P. Graf MD, PhD) Nationale Ethikkommission, Mozartstrasse 21, DE-79104 } \\
\text { Freiburg }\end{array}$ \\
\hline A.Z. Sint-Blasius Vascular Surgery & \\
\hline Imelda Hospital Cardiovascular \& Thoracic Surgery & $\begin{array}{l}\text { Universitair Ziekenhuis Gent, Commissie voor Medische Ethiek, De Pintelaan 185B, BE } \\
9000 \text { Ghent }\end{array}$ \\
\hline \multicolumn{2}{|l|}{ Ghent University Hospital Vascular Surgery } \\
\hline Zol St-Jan & Commissie Medische Ethiek, Schiepse Bos 6, BE-3600 Genk \\
\hline Medical University Graz & Ethikkommission, Univ.Prof. DI Dr. Haas, Aenbruggerplatz 2, A-8036 Graz \\
\hline Luzerner Kantonsspital & $\begin{array}{l}\text { Präsident der Ethik Kommission des Kantons Luzern, Luzerner Kantonspital, } 6000 \\
\text { Lucerne } 16\end{array}$ \\
\hline St. Antonius Hospital & VCMO ST Antoniusziekenhuis, Koekoekslaan 1, 3435 CM Nieuwegein \\
\hline
\end{tabular}


review safety data for subject safety, the study conduct, and progress.

\section{Adverse and serious adverse events}

Adverse events (AEs) are defined as any untoward medical occurrence in a subject whether or not considered related to the study device that is identified or worsens during the trial. AEs are classified following the flowchart below (Figure 2).

AEs will be assessed and documented by the site investigators at the time of the procedure and at all follow-up visits (scheduled and unscheduled). All suspected AEs will be recorded on the AE Log in the electronic clinical report form (e-CRF). An adverse device effect (ADE) is any untoward and unintended response to a medical device including any event resulting from insufficiencies or inadequacies in the instructions for use or the deployment of the device as well as any event that is a result of a user error.

AEs will be classified on the AE Log of the e-CRF as follows, with categories 3 to 5 considered to meet the definition of a serious adverse event (SAE): (1) no therapy, no consequence; (2) nominal therapy, no consequence; (3) required intervention to prevent serious outcome; (4) hospitalization or prolongation of hospitalization; (5) persistent/significant disability/incapacity life-threatening/ death. Major or minor wound debridement is not considered an AE. SAEs include AEs of categories 3-5 and include any untoward medical occurrence in a subject which:

1. Led to a death or

2. led to a serious deterioration in the health of the subject that:

a. resulted in a life-threatening illness or injury, and/or

b. resulted in a permanent impairment of a body structure or body function, and/or

c. required subject hospitalization or prolongation of existing hospitalization and/or

d. resulted in a medical or surgical intervention to prevent permanent impairment to body structure or body function.

A serious adverse device effect (SADE) is an adverse effect that results in any of the consequences characteristic of an SAE or that might have led to any of these consequences had suitable action not been taken, had intervention not been made, or had circumstances been less opportune.

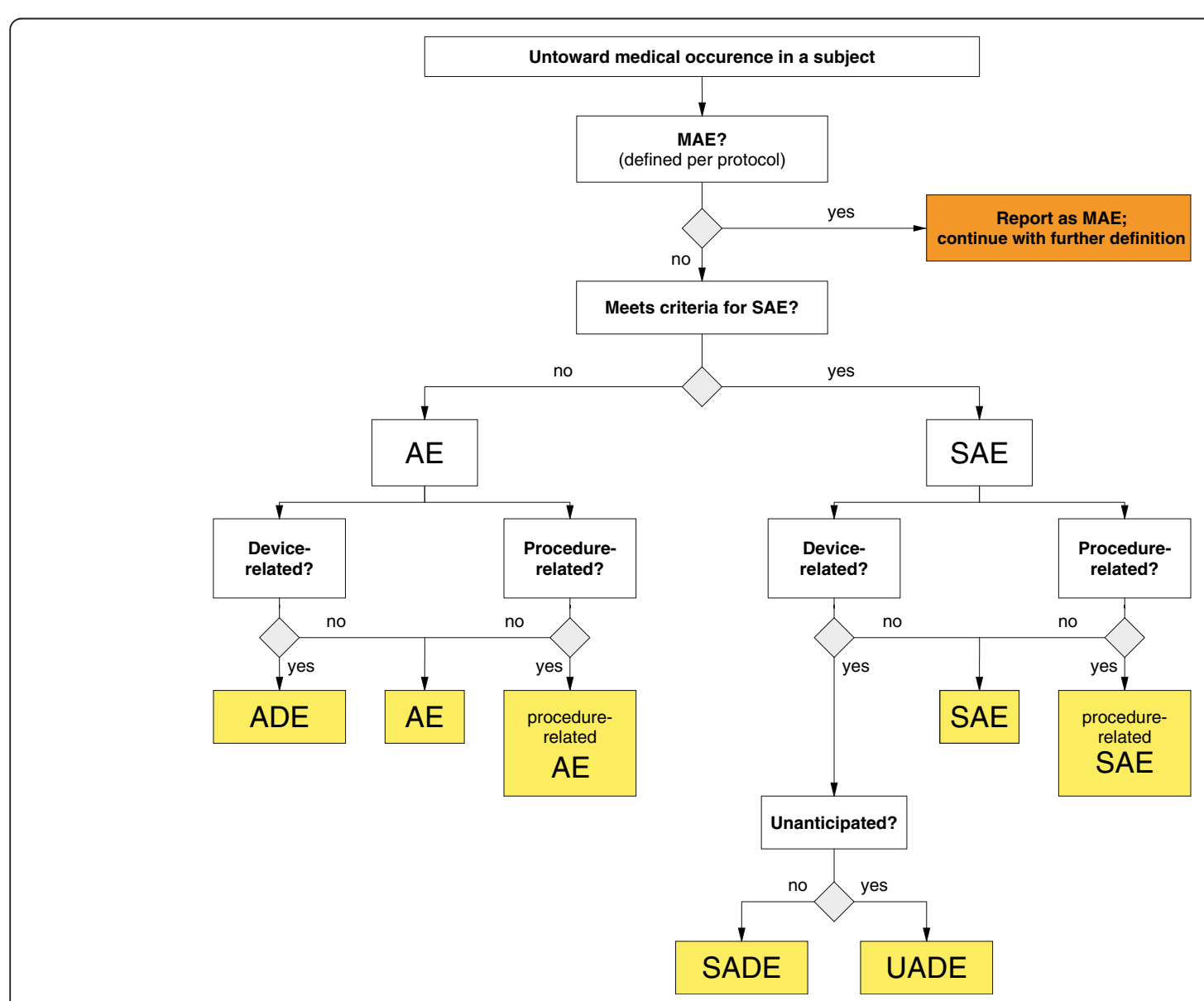

Figure 2 Adverse events categorization flowchart. 
An unanticipated adverse device effect (UADE) is defined as (1) any serious adverse effect on health or safety or any life-threatening problem or death caused by or associated with the use of the investigational device if that effect, problem, or death was not previously identified in nature, severity, or degree of incidence in the investigational plan or application (including a supplementary plan or application) or (2) as any other unanticipated serious problem associated with a device that relates to the rights, safety, or welfare of subjects.

In addition to the "generic" AE Log, dedicated sections are present in the e-CRF allowing tracking of specific events as follows: (1) all TLR; (2) all target extremity revascularization (TER); (3) all deaths; (4) all amputations; (5) all UADEs.

MAEs as in Figure 2 are defined as composite rates of all-cause death, minor amputation, and major amputation.

\section{Statistical analysis}

The total sample size for the IN.PACT DEEP trial was calculated at 357 subjects, which fully powers the primary coefficacy endpoint of LLL (80\%) and the primary composite safety endpoint (80\%) based on initial estimates of event rates and effect sizes of the two arms, IA-DEB and PTA, randomized 2:1. The primary co-efficacy endpoint of 12month TLR is powered to $65 \%$ with the indicated sample size.

\section{Primary co-efficacy endpoints}

The first primary efficacy endpoint is LLL, assessed at 12 months or at the time of TLR; the second is incidence of clinically driven TLR assessed through 12 months. Each will be tested for superiority in a comparison of the randomized groups.

\section{Sample size consideration \\ Primary efficacy endpoint: angiographic cohort}

The statistical hypothesis of superiority is assessed using a two-sample $t$-test of IA-DEB versus PTA, with 5\% two-sided alpha and $80 \%$ desired power. Estimates relevant to the LLL primary efficacy endpoint depend on two observations:

1. The hypothesized treatment effect - that is, the mean difference between the IA-DEB and PTA arms at 12 months.

2. An estimate of variability, specifically the standard deviation (SD).

To obtain estimates of these numbers, a meta-analysis of PTA by Romiti et al. [12] was examined for results on (binary) primary patency. The indicated value was $58.1 \%$ at 12 months, meaning that $58.1 \%$ of enrolled subjects in the studies summarized had binary primary patency (restenosis less than 50\%) at 12 months. The value of $58.1 \%$ for binary patency was used in a simulation to estimate the mean LLL indexed to reference vessel diameter as follows.

1. The standard deviation of indexed LLL was assumed to be 0.25 , which is a reasonably conservative value for a random variable of this type.

2. Indexed LLLs were then simulated as being drawn from a normal distribution such that $58.1 \%$ of the cumulative distribution function lay below 0.5 , the value that defines binary patency. Based upon the parameters above, the mean for the PTA group is estimated to be 0.45 . This is reasonable in that it is somewhat less than 0.5 , which would be expected to be the case since more than half of subjects had binary patency.

Assuming a $30 \%$ relative reduction in mean indexed LLL in the IA-DEB group compared to the PTA group-that is, a mean in the IA-DEB group of $(1-0.3)$ $(0.45)=0.315$-and accounting for $25 \%$ potential endpoint attrition (larger than the $10 \%$ for safety since death is not included in the endpoint and the period of followup is longer), the resulting sample size is 168 (with 112 expected in the IA-DEB group and 56 in the PTA group) subjects in the randomized subgroup qualifying for evaluation of LLL. Note: subjects undergoing bailout or provisional stenting in either randomized arm are considered to continue as normal for endpoint analysis (per ITT principles as below).

\section{Primary efficacy endpoint: clinical cohort}

For the efficacy endpoint of 12-month TLR, the statistical hypothesis is tested using Fisher's exact test of proportions of IA-DEB versus PTA, again with 5\% two-sided alpha and $80 \%$ desired power. For this endpoint, it is assumed that among evaluable subjects (that is, those not lost to followup), 50\% of PTA subjects will undergo TLR during the 12month follow-up and that a relative reduction of $30 \%$ in TLR will be seen in IA-DEB subjects, resulting in a rate of $[(1-0.3)(0.5)=0.35] 35 \%$ for TLR in IA-DEB subjects. After $25 \%$ attrition as before, the required sample size is 504 .

As this sample size is larger than the intended enrollment for the trial, the power for this endpoint in the current trail based upon intended sample size of 357 is $65 \%$ rather than $80 \%$. An interim analysis will be conducted when 150 subjects have attained 12 months of follow-up for the purpose of potentially stopping early for efficacy on this endpoint.

\section{Primary safety endpoint}

The primary safety endpoint for the proposed trial is a composite of all-cause death, major amputation, and 
clinically driven TLR, tested for non-inferiority using Blackwelder's [33] method between the randomized groups and evaluated at the 6-month follow-up visit with a non-inferiority margin defined at $10 \%$. For this endpoint, it is assumed that among evaluable subjects (that is, those not lost to follow-up), $40 \%$ of PTA subjects will experience an event meeting the safety endpoint by the 6-month follow-up and that a relative reduction of $15 \%$ in TLR will be seen in IA-DEB subjects, resulting in a rate of $[(1-0.15)(0.4)=0.34] 34 \%$ in IA-DEB subjects. After $10 \%$ potential attrition relative to this endpoint, the required sample size is 357 (with 238 expected in the IA-DEB group and 119 in the PTA group). The power for this test is greater than $80 \%$.

\section{Early trial termination}

An interim analysis of 150 subjects at 12 months to assess the primary safety and efficacy endpoints is to be performed by an independent group and maintained confidentially. The objective of this interim analysis is two-fold:

- To permit early stopping of the trial for overwhelming efficacy (and not futility)

- For use in designing and powering a subsequent United Stated Investigational Device Exemption (US IDE) trial.

The interim analysis to assess all of the primary safety and efficacy endpoints will be conducted once 150 subjects (approximately 100 IA-DEB and 50 PTA) have reached their 12-month follow-up visits. This interim look would permit early stopping for efficacy using an O'Brien-Fleming-type alpha-spending function. Based on 150 out of 357 total enrolled subjects in the trial, the two-sided $P$-values required at the interim and final looks will be 0.006 and 0.048 , respectively.

Incorporating the Hochberg adjustments, efficacy would be met at the interim look if at least one of the co-primary endpoints is met at a $P$-value of 0.003 or if both are met at 0.006; similarly, at the final look, efficacy will be met if either of the co-primary endpoints is met at a $P$-value of 0.024 or if both are met at 0.048 .

\section{Intervention}

Before the initiation of the interventional procedure, a 55$\mathrm{cm}$ radiopaque ruler was affixed to the index extremity extending from tibial tuberosity to the level of the lateral malleolus. This approach ensured adequate angiographic documentation of the tibial lesion treatment zone and assured appropriate angiographic analysis (i.e., LLL) within the treatment zone on all subsequent planned imaging studies and unplanned interventions. Approach to the tibial artery target lesion was contralateral retrograde or ipsilateral antegrade. Accessing the target lesion (TL) from the popliteal or pedal approach was contraindicated. All imaging was to be performed using digital subtraction angiography. If the subject met the pre-specified angiographic cohort eligibility criteria (Table 1), the subject was selectively entered into the angiographic cohort trial arm.

The treatment objective is to obtain a patent infrapopliteal artery target lesion, with uninterrupted blood flow to the foot. Therefore, significant lesions in the aortoiliac and/or femoropopliteal arterial segments were treated during the index procedure. However, treatment success of the inflow lesion was to be documented as successful (i.e., residual stenosis $<30 \%$ by visual estimate). This interventional strategy conforms to standard of practice in maximizing inflow into the infrapopliteal segment in CLI patients.

Prior to intervention, digital subtraction angiography of the inflow segment and pedal runoff imaging were required. Standard interventional techniques in crossing arterial stenoses and/or occlusions were to be employed; re-entry devices in the infrapopliteal segment were contraindicated. Pre-dilatation with an undersized (nondrug-eluting) balloon were required in case of total occlusions and sub-occlusive lesions; however, adjuncts to angioplasty, including atherectomy, cutting or scoring balloons were not permitted. The subject was randomized to treatment with the IA-DEB or PTA only after successful traversal of the TL and placement of the guidewire into the distal true lumen. If all requirements were not documented, the subject was considered a screen failure.

After documentation of the above requirements, if the subject was randomized to the investigational treatment, the IA-DEB balloon of a diameter approximating a 1:1 ratio to the reference artery diameter was deployed and inflated for a minimum of $1 \mathrm{~min}$. If deemed necessary by the operator, a post-dilatation of the TL was performed using a standard short-length standard PTA balloon intended to resolve persistent residual stenosis.

Provisional stenting was permitted according to the following specific criteria:

a) Major dissection (grade $\mathrm{C}$ or above). However, in order to limit stenting as much as possible, prolonged standard PTA (3 $\mathrm{min})$ with a non-drugeluting balloon was to be performed first.

b) Occlusive complication (e.g., recoil) that resulted in severely decreased target vessel flow that did not respond to repeated prolonged balloon inflation.

c) Persistent residual stenosis $>50 \%$ (documented by the worst of 2 orthogonal views after optimal PTA).

In the case of provisional stent implantation, a commercially available bare metal stent could be used. As 
Table 4 Trial assessment requirements

Baseline Procedure Discharge

30 days

3 months

6 months 1 year 2 years

$\begin{array}{ccc}3 \text { years } & 4 \text { years } & 5 \text { years } \\ (1065-1125) & (1430-1490) & (1795-185\end{array}$

5) Unsched Demography $x$

Medical History

Physical Exam

Concomitant Meds

Anticoagulant/antiplatelet therapy

Informed Consent

Incl/Excl Evaluation

Routine Lab testing (see Table 2)

Ankle pressure, toe pressure, $\mathrm{TCPO}_{2}, \mathrm{PVR}$ (at least 1 required) and brachial pressure

Wound assessment ${ }^{7}$ and Wound Care

Rutherford Staging

EQ5D

(24-45)

(84-105)(84-105)

$x$

$\begin{array}{cccc}x & & x & x \\ x^{1} & x^{2} & x & x \\ x & & & \\ x & x & x & x \\ x^{3} & & x & x \\ x^{3} & & x^{8} & x \\ x & & & x \\ x & & & \end{array}$

WIQ

Angiography

Hospital FU Visits

$x^{3}$

Telephone FU

$x^{3}$

$x$

Adverse Event Assessment

${ }^{1}$ Aspirin $(100 \mathrm{mg})$ at least 4 days prior to the index intervention, alternatively at least $500 \mathrm{mg}$ loading dose prior to or within 2 hours post procedure; Clopidogrel $75 \mathrm{mg}$ daily at least 4 days prior to the index intervention, alternatively at least $300 \mathrm{mg}$ loading dose prior to or within 2 hours post procedure (or ticlopidine, if required); the use of bivalirudin (Angiox ${ }^{\mathrm{TM}}$ ) was allowed as an alternative to heparin.

${ }_{2}^{2}$ Following the PTA prosedure, subjects were to be prescribed daily a Prolonged antiplatelet therapy could be given at the discretion of the physician and should be considered after placement of stents.

Prolonged antiplatelet therapy
${ }^{3}$ Within 6 weeks of procedure.

${ }^{3}$ Within 6 weeks of procedure.
${ }^{4}$ For Angio Sub-group subjects only.

${ }^{4}$ For Angio Sub-group subjects only.
If subject undergoes an unscheduled angiogram; a copy of the angiogram must be forwarded to the Angiographic Core Lab for adjudication.

${ }^{7}$ Wound assessment performed via the SilhouetteMobile.

${ }^{8}$ Wound assessment
Onound Care. 
this is an ITT analysis, subjects receiving provisional stents will be tracked in their respective assigned randomized trial arms. A completion digital subtraction angiogram in two oblique views of the TL and pedal run-off was required.

\section{Follow-up}

Clinical follow-up will proceed as noted in Table 4. All subjects will be followed for 5 years.

\section{Data collection}

Data will be collected via e-CRF during treatment at all investigational centers and will be completed prospectively during the hospital admission and follow-up.

\section{Study device}

IA-DEB manufactured by Medtronic (Santa Rosa, CA, USA) features a 0.014" guidewire-compatible, over-thewire drug-eluting balloon that uses paclitaxel as the therapeutic agent and urea as excipient. The characteristics and mode of action of the IN.PACT drug-eluting balloon have been described elsewhere [17,34,35].

\section{Discussion}

While the body of clinical experience involving the treatment of CLI continues to evolve rapidly, our knowledge and understanding of the many factors that impact treatment outcomes of this heterogeneous and complex disease state remain incomplete. Nevertheless, there have been important recent contributions to our appreciation of factors affecting clinical outcomes, including the concept of angiosome-directed revascularization and recognition of the importance of restoring patency of the pedal run-off vessels. New technology has also provided a potential improvement over current modalities of therapy. A substantial treatment effect of the IA-DEB technology in improving infrapopliteal vessel patency and reducing re-intervention rates when compared to standard PTA has been reported at 3-, 6-, and 12-month time points by single-center registries $[10]$ and randomized trials $[18,19]$.

The reduction in re-intervention rates in this challenging CLI population is an undisputable hallmark of successful IA-DEB use; however, the important "patient-centric" outcomes of limb salvage, pain relief, mobility restoration, and improvement in quality of life remain equally important goals and endpoints in both clinical practice and research. Unfortunately, these fundamental "patient-centric" endpoints and essential post-treatment care and follow-up are infrequently pre-specified and/or are incompletely monitored and reported in CLI trials. Nevertheless, the three IADEB studies cited above reinforce the nature of the challenges in establishing a clear correlation between improved vessel patency and "patient-centric" outcomes. They also emphasize that successful CLI care goes beyond promoting durable vessel patency and is but one essential aspect of a multidisciplinary team approach, an approach that is representative of the IN.PACT DEEP trial design.

Therefore, as our clinical experience in the multifaceted care and treatment of CLI patients evolves, the IN.PACT DEEP stands as a unique milestone robust trial, which will contribute to our fundamental understanding of the role of durable vessel patency and its influence on "patient-centric" outcomes targeting the full spectrum of Rutherford class 45-6 critical limb ischemia and the identification of clinical and anatomical profiles that will derive the optimal benefit from DEB therapy.

\section{Trial status}

In.PACT DEEP completed subject enrollment/randomization in July 2012, and the final enrolled subjects returned for clinical follow-up in August 2013. Public presentation of the trial co-primary and secondary endpoints is expected in 2014.

\section{Conclusion}

The IN.PACT DEEP represents the first completed, largest, prospective, multicenter, randomized, controlled comparison of plain old balloon angioplasty to drug-eluting balloon angioplasty in infrapopliteal arteries of patients with CLI. The trial design is rigorous and provides unbiased assessment of outcomes through independent angiographic and wound-healing core labs and clinical event committee adjudication. IN.PACT DEEP tests the hypothesis that the IN. PACT Amphirion paclitaxel drug-eluting balloon provides superior 1-year vessel patency, assessed by late lumen loss (LLL), and whether any potential improvement in vessel patency is associated with a significant reduction in clinically driven target lesion revascularization through 1 year. Clinical follow-up is planned through 5 years and will continue to yield important information regarding the long-term impact of these two interventional strategies and set a new standard for clinical evidence that will assist physicians and caregivers with important direction in the care of this complex and challenging patient cohort.

\section{Abbreviations \\ ADE: Adverse device effect; AE: Adverse event; AFS: Amputation-free survival; Angio-TL: Angiographic target lesion; CEC: Clinical events committee; CLI: Critical limb ischemia; DEB: Drug-eluting balloon; DSMB: Data safety monitoring board; e-CRF: electronic clinical report form; IA-DEB: IN.PACT Amphirion drug-eluting balloon; ITT: Intention to treat; LLL: Late lumen loss; MAE: Major adverse event; PTA: Percutaneous transluminal angioplasty; QoL: Quality of life; SADE: Serious adverse device effect; SAE: Serious adverse event; TER: Target extremity revascularization; TL: Target lesion; TLR: Target lesion revascularization; UADE: Unanticipated adverse device effect; US IDE: United States investigational device exemption; WRA: Wound-related artery.}

\section{Competing interests}

AM and TZ are consultants for Medtronic; FM receives grant support from Medtronic. DS and ML are Medtronic employees. All other authors have nothing to disclose. 


\section{Authors' contributions}

$T Z, I B, D S$, and $M L$ conceived and participated in the design of the study. $M B R, M B O, A M, P P$, and FV contributed to the design of the study. ML drafted the manuscript. All authors approved the final manuscript.

\section{Acknowledgements}

The authors wish to acknowledge the efforts of Krishna Rocha-Singh, MD, for his input and critique of the trial design and review of the manuscript; Martin Faiss, PhD, for his critical review of the manuscript, and the assistance of Judith Greengard, PhD, RAC, in its preparation.

\section{Funding}

Medtronic, Inc. (Santa Rosa, CA, USA) funded the IN.PACT DEEP Trial.

\section{Author details}

${ }^{1}$ Department of Angiology, Universitäts Herzzentrum Freiburg Bad Krozingen, Bad Krozingen, Germany. 'Swiss Cardiovascular Center, Division of Angiology, University Hospital, Inselspital, Bern, Switzerland. ${ }^{3}$ Center of Vascular Medicine, Park Hospital Leipzig, Leipzig, Germany. ${ }^{4}$ Department of Angiology, Medical University Graz, Graz, Austria. ${ }^{5}$ Department of Vascular Surgery, A.Z. Sint-Blasius, Dendermonde, Belgium. ${ }^{6}$ Invasive Cardioangiology GVM Care and Research, Palermo, Italy. ${ }^{7}$ Department of Cardiovascular \& Thoracic Surgery, Imelda Hospital, Bonheiden, Belgium. ${ }^{8}$ Department of Vascular Surgery, Ghent University Hospital, Ghent, Belgium. ${ }^{9}$ Department of Medical Affairs, Endovascular Therapies, Medtronic Cardiovascular, Santa Rosa CA, USA.

Received: 16 July 2013 Accepted: 28 January 2014 Published: 19 February 2014

\section{References}

1. Beard JD: Which is the best revascularization for critical limb ischemia: endovascular or open surgery? J Vasc Surg 2008, 48:112-116S.

2. Blevins WA Jr, Schneider PA: Endovascular management of critical limb ischemia. Eur J Vasc Endovasc Surg 2010, 39(6):756-761.

3. Adam DJ, Beard JD, Cleveland T, Bell J, Bradbury AW, Forbes JF, Fowkes FG, Gillepsie I, Ruckley CV, Raab G, Storkey H: BASIL trial participants. Bypass versus angioplasty in severe ischaemia of the leg (BASIL): multicenter, randomised controlled trial. Lancet 2005, 366(9501):1925-1934

4. Conrad MF, Kang J, Cambria RP, Brewster DC, Watkins MT, Kwolek CJ, LaMuraglia GM: Infrapopliteal balloon angioplasty for the treatment of chronic occlusive disease. J Vasc Surg 2009, 50(4):799-805.

5. Söderström M, Arvela $E$, Venermo $M$, Lepäntalo $M$, Albäck A: Tertiary patency as a measure of active revascularization policy for leg salvage. Ann Vasc Surg 2011, 25(2):159-164.

6. Kudo T, Chandra FA, Ahn SS: The effectiveness of percutaneous transluminal angioplasty for the treatment of critical limb ischemia: a 10-year experience. J Vasc Surg 2005, 41(3):423-435.

7. Haider SN, Kavanagh EG, Forlee M, Colgan MP, Madhavan P, Moore DJ, Shanik GD: Two-year outcome with preferential use of infrainguinal angioplasty for critical ischemia. J Vasc Surg 2006, 43:504-512.

8. Graziani L, Silvestro A, Bertone V, Manara E, Andreini R, Sigala A, Mingardi R, De Giglio R: Vascular involvement in diabetic subjects with ischemic foot ulcer: a new morphologic categorization of disease severity. Eur J Vasc Endovasc Surg 2007, 33(4):453-460.

9. Parsons RE, Suggs WD, Lee JJ, Sanchez LA, Lyon RT, Veigh FJ: Percutaneous transluminal angioplasty for the treatment of limb threatening ischemia: do the results justify an attempt before bypass grafting? J Vasc Surg 1998, 28:1066-1071.

10. Schmidt A, Ulrich M, Winkler B, Klaeffling C, Bausback Y, Bräunlich S, Botsios S, Kruse HJ, Varcoe RL, Kum S, Scheinert D: Angiographic patency and clinical outcome after balloon-angioplasty for extensive infrapopliteal arterial disease. Catheter Cardiovasc Interv 2010, 76(7):1047-1054.

11. Baumann F, Willenberg T, Do DD, Keo HH, Baumgartner I, Diehm N: Endovascular revascularization of below-the-knee arteries: prospective short-term angiographic and clinical follow-up. J Vasc Interv Radiol 2011, 22(12):1073-1665.

12. Romiti M, Albers M, Brochado-Neto FC, Durazzo AE, Pereira CA, De Luccia N: Meta-analysis of infrapopliteal angioplasty for chronic critical limb ischemia. J Vasc Surg 2008, 47(5):975-981.
13. Adam DJ, Bradbury AW: TASC II document on the management of peripheral arterial disease. Eur J Vasc Endovasc Surg 2007, 33(1):1-2.

14. Hirsch AT, Haskal ZJ, Hertzer NR, Bakal CW, Creager MA, Halperin JL, Hiratzka LF, Murphy WR, Olin JW, Puschett JB, Rosenfield KA, Sacks D, Stanley JC, Taylor LM Jr, White CJ, White J, White RA, Antman EM, Smith SC Jr, Adams CD, Anderson JL, Faxon DP, Fuster V, Gibbons RJ, Hunt SA, Jacobs AK, Nishimura R, Ornato JP, Page RL, Riegel B, et al: ACC/AHA 2005 Practice Guidelines for the management of patients with peripheral arterial disease (lower extremity, renal, mesenteric, and abdominal aortic): a collaborative report from the American Association for Vascular Surgery/ Society for Vascular Surgery, Society for Cardiovascular Angiography and Interventions, Society for Vascular Medicine and Biology, Society of Interventional Radiology, and the ACC/AHA Task Force on Practice Guidelines (Writing Committee to Develop Guidelines for the Management of Patients With Peripheral Arterial Disease): endorsed by the American Association of Cardiovascular and Pulmonary Rehabilitation; National Heart, Lung, and Blood Institute; Society for Vascular Nursing; TransAtlantic Inter-Society Consensus; and Vascular Disease Foundation. Circulation 2006, 113(11):e463-e654.

15. Conte MS: Diabetic revascularization: endovascular versus open bypassdo we have the answer? Semin Vasc Surg 2012, 25(2):108-114.

16. Nolan BW, De Martino RR, Stone DH, Schanzer A, Goodney PP, Walsh DW, Cronenwett JL, Vascular Study Group of New England: Prior failed ipsilateral percutaneous endovascular intervention in patients with critical limb ischemia predicts poor outcome after lower extremity bypass. J Vasc Surg 2011, 54(3):730-735.

17. Schmidt A, Piorkowski M, Werner M, Ulrich M, Bausback $Y$, Bräunlich S, Ick H, Schuster J, Botsios S, Kruse HJ, Varcoe RL, Scheinert D: First experience with drug-eluting balloons in infrapopliteal arteries: restenosis rate and clinical outcome. J Am Coll Cardiol 2011, 58(11):1105-1109.

18. Fanelli F, Cannavale A, Boatta E, Corona M, Lucatelli P, Wlderk A, Cirelli C, Salvatori FM: Lower limb multilevel treatment with drug-eluting balloons: 6-month results from the DEBELLUM randomized trial. $J$ Endovasc Ther 2012, 19(5):571-580

19. Liistro F, Porto I, Angioli P, Grotti S, Ricci L, Ducci K, Falsini G, Ventoruzzo G, Turini F, Bellandi G, Bolognese L: Drug-eluting balloon in peripherAl inTErvention for below the knee angioplasty evaluation (DEBATE-BTK): a randomized trial in diabetic patients with critical limb ischemia. Circulation 2013: [Epub ahead of print].

20. Tepe G, Zeller T, Albrecht T, Heller S, Schwarzwälder U, Beregi JP, Claussen CD, Oldenburg A, Scheller B, Speck U: Local delivery of paclitaxel to inhibit restenosis during angioplasty of the leg. N Engl J Med 2008, 358(7):689-699.

21. Werk M, Langner S, Reinkensmeier B, Boettcher HF, Tepe G, Dietz U, Hosten $N$, Hamm B, Speck U, Ricke J: Inhibition of restenosis in femoropopliteal arteries: paclitaxel-coated versus uncoated balloon: femoral paclitaxel randomized pilot trial. Circulation 2008, 118(13):1358-1365.

22. Werk M, Albrecht T, Meyer DR, Ahmed MN, Behne A, Dietz U, Eschenbach G, Hartmann H, Lange C, Schnorr B, Stiepani H, Zoccai GB, Hänninen EL: Paclitaxel-coated balloons reduce restenosis after femoro-popliteal angioplasty: evidence from the randomized PACIFIER trial. Circ Cardiovasc Interv 2012, 5(6):831-840.

23. Micari A, Cioppa A, Vadalà G, Castriota F, Liso A, Marchese A, Grattoni C, Pantaleo P, Cremonesi A, Rubino P, Biamino G: 2-year results of paclitaxeleluting balloons for femoropopliteal artery disease: evidence from a multicenter registry. JACC Cardiovasc Interv 2013, 6(3):282-289.

24. Taylor G, Palmer J: The vascular territories (angiosomes) of the body: experimental studies and clinical application. Br J Plast Surg 1987, 40:113-141.

25. lida O, Nanto S, Uematsu M, Ikeoka K, Okamoto S, Dohi T, Fujita M, Terashi $H$, Nagata S: Importance of the angiosome concept for endovascular therapy in patients with critical limb ischemia. Catheter Cardiovasc Interv 2010, 75(6):830-836.

26. Kabra A, Suresh K, Vivekanand V, Vishnu M, Sumanth $R$, Nekkanti M: Outcomes of angiosome and non-angiosome targeted revascularization in critical lower limb ischemia. J Vasc Surg 2013, 57:44-49.

27. Neville RF, Attinger CE, Bulan EJ, Ducic I, Thomassen M, Sidawy AN: Revascularization of a specific angiosome for limb salvage: does the target artery matter? Ann Vasc Surg 2009, 23(3):367-373.

28. Peregrin JH, Koznar B, Kovác J, Lastovicková J, Novotný J, Vedlich D, Skibová J: PTA of infrapopliteal arteries: long-term clinical follow-up and analysis 
of factors influencing clinical outcome. Cardiovasc Intervent Radiol 2010, 33(4):720-725.

29. Faglia E, Clerici G, Clerissi J, Mantero M, Caminiti M, Quarantiello A, Curci V, Lupattelli T, Morabito A: When is a technically successful peripheral angioplasty effective in preventing above-the-ankle amputation in diabetic patients with critical limb ischaemia? Diabet Med 2007, 24(8):823-829.

30. Manzi M, Cester G, Palena LM, Alek J, Candeo A, Ferraresi R: Vascular imaging of the foot: the first step toward endovascular recanalization. Radiographics 2011, 31(6):1623-1636.

31. Manzi M, Fusaro M, Ceccacci T, Erente G, Dalla Paola L, Brocco E: Clinical results of below-the knee intervention using pedal-plantar loop technique for the revascularization of foot arteries. I Cardiovasc Surg (Torino) 2009, 50(3):331-337.

32. Utsunomiya M, Nakamura M, Nakanishi M, Takagi T, Hara H, Onishi K, Yamada T, Sugi K: Impact of wound blush as an angiographic end point of endovascular therapy for patients with critical limb ischemia. J Vasc Surg 2012, 55(1):113-1121.

33. Blackwelder W: "Proving the null hypothesis" in clinical trials. Control Clin Trials 1982, 3:345-353.

34. Kelsch B, Scheller B, Biedermann M, Clever YP, Schaffner S, Mahnkopf D, Speck U, Cremers B: Dose response to Paclitaxel-coated balloon catheters in the porcine coronary overstretch and stent implantation model. Invest Radiol 2011, 46(4):255-263.

35. Schnorr B, Kelsch B, Cremers B, Clever YP, Speck U, Scheller B: Paclitaxelcoated balloons - Survey of preclinical data. Minerva Cardioangiol 2010, 58(5):567-582.

doi:10.1186/1745-6215-15-63

Cite this article as: Zeller et al.: IN.PACT Amphirion paclitaxel eluting balloon versus standard percutaneous transluminal angioplasty for infrapopliteal revascularization of critical limb ischemia: rationale and protocol for an ongoing randomized controlled trial. Trials 2014 15:63.

\section{Submit your next manuscript to BioMed Central and take full advantage of:}

- Convenient online submission

- Thorough peer review

- No space constraints or color figure charges

- Immediate publication on acceptance

- Inclusion in PubMed, CAS, Scopus and Google Scholar

- Research which is freely available for redistribution

Submit your manuscript at www.biomedcentral.com/submit
C Biomed Central 